\title{
Towards a Platform to evaluate the impact of Resource Information Distribution in IoT Environments ${ }^{\star}$
}

\author{
Paula Verghelet ${ }^{10000-0003-3861-2902]}$ and Esteban \\ Mocskos $^{1,2}[0000-0002-6473-7672]$ \\ 1 Departamento de Computación, \\ Facultad de Ciencias Exactas y Naturales, Universidad de Buenos Aires \\ Buenos Aires (C1428EGA), Argentina. \\ 2 Centro de Simulación Computacional p/Aplic. Tecnológicas/CSC-CONICET, \\ Godoy Cruz 2390, Buenos Aires (C1425FQD), Argentina. \\ \{pverghelet, emocskos\}@dc.uba.ar
}

\begin{abstract}
Internet of Things (IoT) is a paradigm in which every object has the capacity of communicating through the Internet. Cloud Computing is designed to provide computational resources to costumers geographically distributed following an elastic payment strategy. Fog/Edge Computing aims to decrease bandwidth usage keeping the computation near the source of data and avoiding the collapse of network infrastructure when moving all the data from the edge to the cloud data centers. Fog and Cloud environments define a large scale distributed system composed of heterogeneous resources, which has huge theoretical computing power. But using these computational resources poses challenges to distributed applications and scheduling policies.

In this work [1], we show the initial steps to develop a tool to support evaluate the impact of resource information quality to guide scheduling policies. This tool combines simulation and validation and simplifies the deployment of experiments on both sides. The evaluation of this initial proof of concept consists of the deployment of experiments with a different number of devices in a single site and in three different sites across France. Our results show that both simulation and validation platforms present good agreement.
\end{abstract}

Keywords: Resource Information - Internet of Things - Validation · Complex Distributed Systems.

\section{References}

1. Verghelet, Paula and Mocskos, Esteban. "Towards a Platform to Evaluate the Impact of Resource Information Distribution in IoT Environments". In Proceedings of

\footnotetext{
* This work is supported by UBA (UBACyT 20020130200096BA), CONICET (PIO13320150100020CO), and ANPCyT (PICT-2015-2761 and PICT-2015-0370). We thank the access to FIT IoT-LAB infrastructure.
} 
Latin American High Performance Computing Conference, pp. 215-229. Springer, Cham, 2019. 